\title{
Hot-Carrier Extraction in Nanowire-Nanoantenna Photovoltaic Devices
}

\author{
I-Ju Chen ${ }^{1}$, Steven Limpert ${ }^{1}$, Wondwosen Metaferia ${ }^{1,2}$, Claes Thelander ${ }^{1}$, Lars Samuelson ${ }^{1}$, Federico Capasso ${ }^{3}$, \\ Adam M. Burke ${ }^{1}$, Heiner Linke ${ }^{1}$
}

${ }^{1}$ NanoLund and Solid State Physics, Lund University, Box 118, SE-22100 Lund, Sweden

${ }^{2}$ National Renewable Energy Laboratory, Golden, CO 80401, United States

${ }^{3}$ Harvard John A. Paulson School of Engineering and Applied Sciences, Harvard University, Cambridge, Massachusetts 02138, United States

\section{Supporting information:}

\section{Isc measurement for additional devices}

In this section we present photocurrent measurements for additional devices. Also in these devices, we observed a significant increase in $I_{\mathrm{SC}}$ under TE-polarized light after adding nanoantennas (Fig. S1) similar to device I shown in the letter. In Fig. S1, the current polarity corresponds to measurement circuitries with the ammeter on the same side as the nanoantennas relative to the InP barrier as shown in Fig. S1 (c, d). Therefore, the positive sign of $I_{\mathrm{SC}}$ in Fig. S1 $(a, b)$ shows a net flow of electron from the antenna to the barrier.

(a)

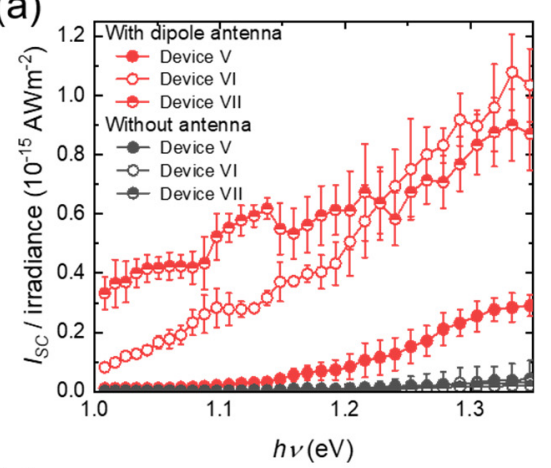

(c)

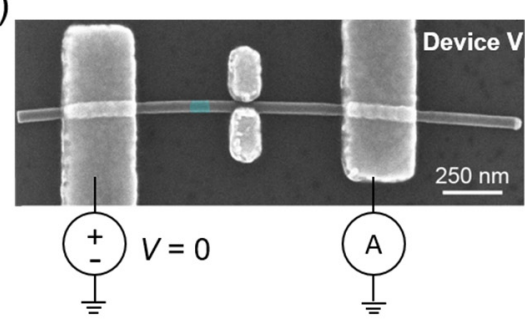

(b)

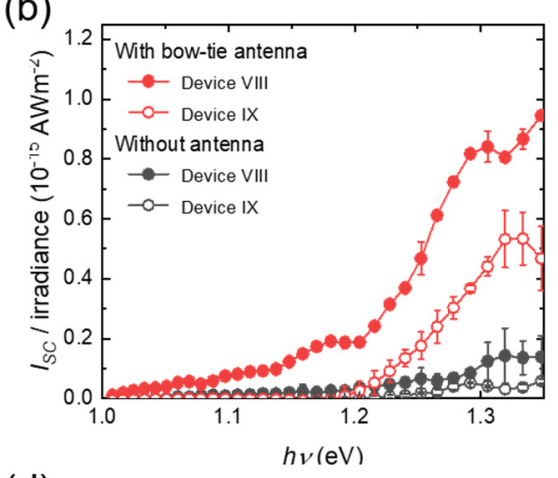

(d)

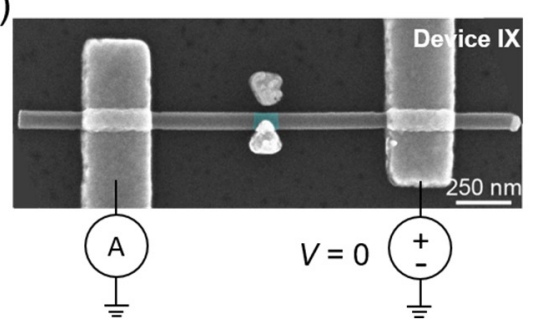

Figure S1. (a, b) Measured $I_{\mathrm{SC}}$ as a function of photon energy $h v$ under TE $(\vec{E} \perp \mathrm{NW})$ polarized light. $I_{\mathrm{SC}}$ was measured before and after the (a) dipole and (b) bow-tie nanoantennas were added. ISC increased significantly after the nanoantennas were added. The SEM image of one of the devices with (c) dipole nanoantenna (Device V) and (d) bow-tie nanoantenna (Device IX) are shown. The measurement circuitries shown in $(c, d)$ are used so that a positive current corresponds to a net flow of electrons from the nanoantenna towards the barrier.

A more detailed analysis is performed for Device II and IV. Figure S2 shows the device structures (Fig. S2(a,c)), FDTD simulations (Fig. S2(b,d)), and ISC measured under TE-polarized light (Fig. S2(e,f)) of Device II and IV. The measured $I_{\mathrm{SC}}$ also shows a clear increase after nanoantennas are added. Theoretical fittings based on the internal photoemission model and FDTD simulations show good agreements with the experimental data. Based on the fittings, we estimate the inelastic scattering lengths to be $30.0 \pm 2.3$ and $34.8 \pm 3.0 \mathrm{~nm}$ and internal quantum efficiencies to be 0.56 and $1.23 \%$ for Devices II and IV, respectively.

For Device II, in contrast to device I shown in the main text, $I_{\mathrm{SC}}$ has the same polarity before and after the nanoantenna is added. It indicates that light absorptions outside the nanoantenna region contribute to $I_{\mathrm{SC}}$ in the 
same direction as light absorption near the gap of the nanoantenna. This is consistent with the observation that Device II shows a $V_{\mathrm{OC}}$ very close to $V_{\mathrm{OC} \text {,max }}$ up to $h v>1.2 \mathrm{eV}$.
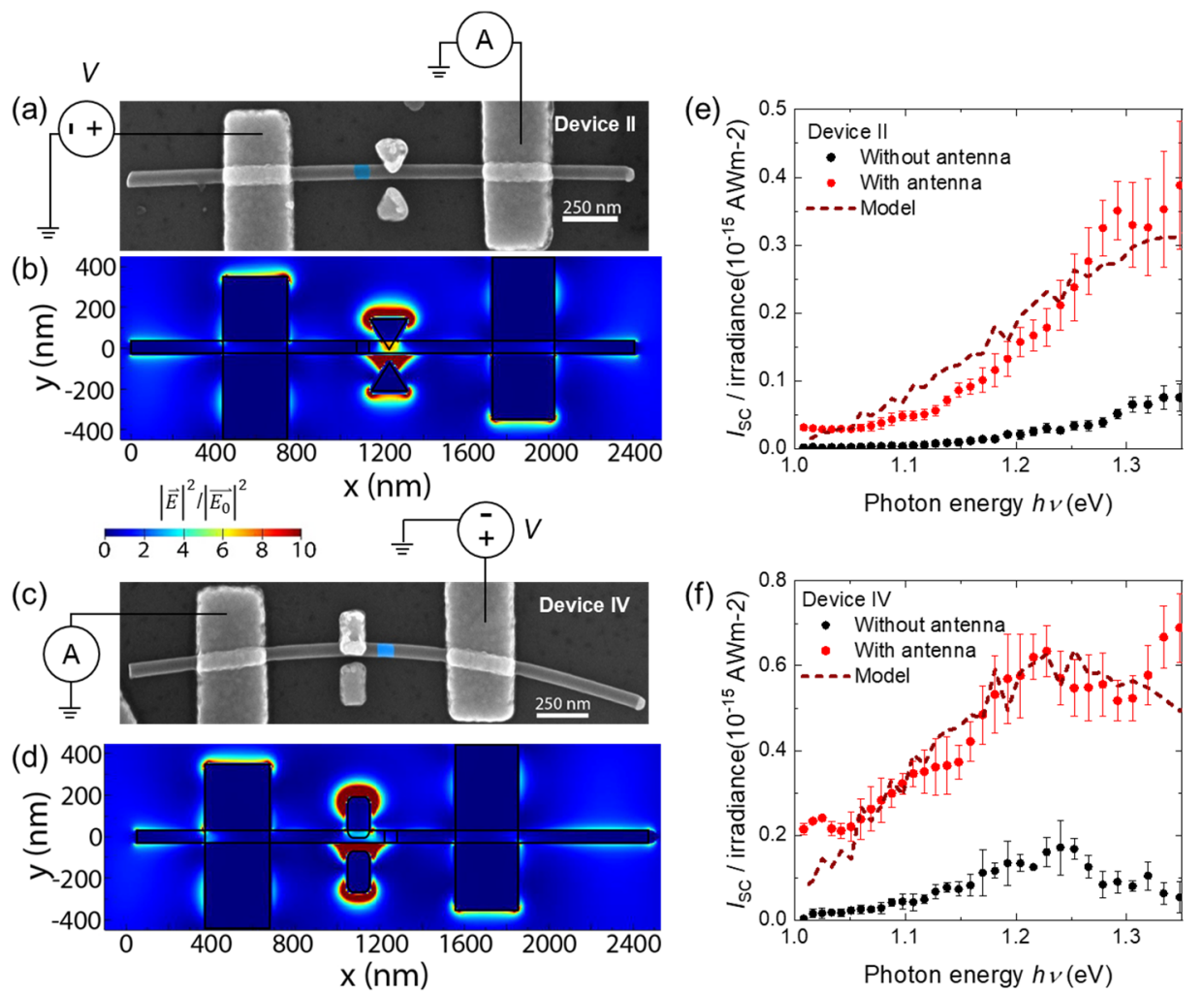

Figure S2. (a,c) SEM images of Devices II and IV. The InP segments are false colored in blue. (b,d) FDTD simulation of the the normalized near-field intensity $|\vec{E}|^{2} /\left|\overrightarrow{E_{0}}\right|^{2}$ in the device. (e,f) $I_{\mathrm{SC}}$ per irradiance measured in Devices II and IV both before and after the nanoantenns are added. Quantitative agreements are found between $I_{\mathrm{SC}}$ per irradiance as a function of $h v$ and the theoretical fittings based on the internal photoemission model and FDTD simulations.

\section{Spatial dependence of absorption efficiency}

Based on the device structures, the absorption efficiency along the nanowire axis before and after the nanoantennas were added was simulated with the FDTD methods. The results show that outside the nanoantenna region, light is mainly absorbed near the interface between the nanowire and the source/ drain contact. The absorption efficiency profile was used as an input for the three-step internal photoemission model in Eq. 3.

(a)
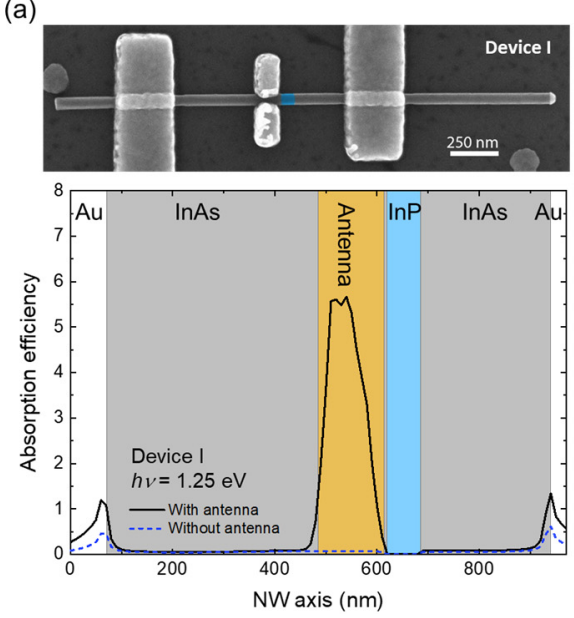

(b)
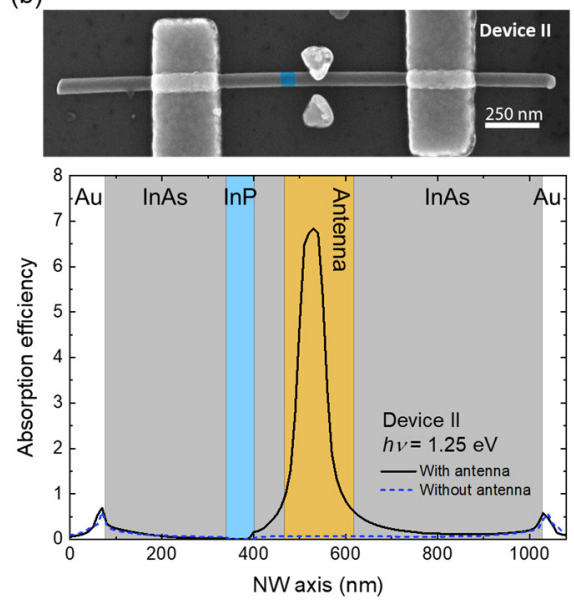
Figure S3. SEM image and the local absorption efficiency under TE-polarized light with $h v=1.25 \mathrm{eV}$ as a function of the spatial coordinate along the nanowire axis in (a) Device I and (b) Device II before and after the nanoantennas were added.

\section{Barrier transmission probability in nanowires}

In this section, we will describe the model used to calculate the energy-dependent probability for electrons in an InAs nanowire to transmit an InP energy barrier. In our model, we adopt the effective mass approximation and a simplified band alignment as shown in Fig. S4(a), and consider the electron transport to be coherent across the barrier.

In a nanowire, due to lateral quantum confinement, the conduction band is composed of 1D subbands, each with a quantum confinement energy $\epsilon_{n}$ (obtained by solving 2D Schrödinger equation) as shown in Fig. S4(b). An electron with energy $E$ can occupy subbands with $\epsilon_{n} \leq E$. For electrons in each 1D subband, their transmission and reflection probability at the InP barrier can be obtained by solving the 1D time-independent Schrödinger equation

$-\frac{d}{d x}\left(\frac{1}{m^{*}} \frac{d \psi(x)}{d x}\right)+V(x) \psi(x)=E \psi(x)$,

with effective mass $m^{*}$ and potential $V(x)$. The wave functions in InAs and InP can be expressed as $(1,2)$

$\psi(x)=\left\{\begin{array}{c}a \exp \left(i k_{1} x\right)+b \exp \left(-i k_{1} x\right),(x<0) \\ c \exp \left(i k_{2} x\right)+d \exp \left(-i k_{2} x\right),(0<x<L) . \\ e \exp \left(i k_{1} x\right),(x>L)\end{array}\right.$

Due to energy conservation the wave numbers $k_{1}$ and $k_{2}$ need to satisfy

$E=\frac{\hbar^{2} k_{1}^{2}}{2 m_{\mathrm{InAs}}}+\epsilon_{n, \mathrm{InAs}}=\frac{\hbar^{2} k_{2}^{2}}{2 m_{\mathrm{InP}}}+\epsilon_{n, \mathrm{InP}}+V_{0}$.

Here we make the critical assumption that the lateral momentum $k_{\perp}$ is conserved as the electrons transmit from InAs to InP. Therefore their quantum confinement energy can be expressed as $\epsilon_{n, \operatorname{InAs}}=\frac{\hbar^{2} k_{\perp}^{2}}{2 m_{\operatorname{InAs}}}$ and $\epsilon_{n, \operatorname{InP}}=\frac{\hbar^{2} k_{\perp}^{2}}{2 m_{\operatorname{InP}}}$, respectively. Electron effective masses in InAs and InP $m_{\mathrm{InAs}}=0.0370 m_{\mathrm{e}}$ and $m_{\mathrm{InP}}=0.0947 m_{\mathrm{e}}(3)$ are used for the calculations here. Based on Eq. S1 and the continuity requirement of $\psi$ and $\frac{1}{m^{*}} \frac{d \psi}{d z}$ at $x=0$ and $L(1)$, the transmission probability for electrons in the nth subband can be obtained.

$T_{n}(E)=\left[1+\left(\frac{v_{1}^{2}-v_{2}^{2}}{2 v_{1} v_{2}}\right)^{2} \sin ^{2} k_{2} L\right]^{-1}$ for $E>V_{0}+\epsilon_{n, \mathrm{InP}}$,

with $v_{1}=k_{1} / m_{1}, v_{2}=k_{2} / m_{2}$.

$T_{n}(E)=\left[1+\left(\frac{v_{1}^{2}-v_{2}^{2}}{2 v_{1} v_{2}}\right)^{2} \sinh ^{2} \kappa_{2} L\right]^{-1}$ for $E<V_{0}+\epsilon_{n, \mathrm{InP}}$,

with $\kappa_{2}=\sqrt{2 m_{2}\left(V_{0}+\epsilon_{n, \operatorname{InP}}-E\right)} / \hbar$ and $v_{2}=\kappa_{2} / m_{2}$. Finally, the overall transmission probability can be obtained from the average of the occupied subbands with

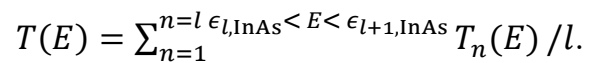

The calculated $T_{1}$ and $T$ are shown in Fig. S4(c). It can be observed that $T_{\mathrm{n}}(E)$ for an individual subband shows a significant over-the-barrier resonance. Yet, because InAs and InP have different effective masses and thus different quantum confinement energies, the resonances largely cancel out when all the subbands are taken into consideration. 
(a)

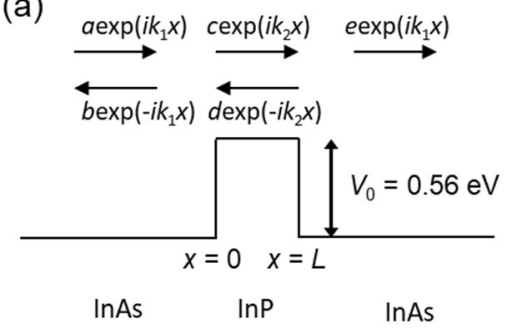

(b)

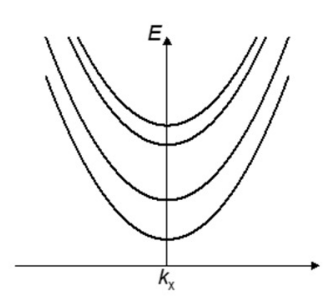

(c)

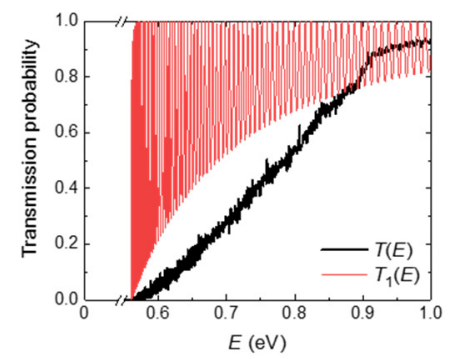

Figure S4. (a) Schematic illustration of incident, reflected, and transmitted electron waves around a square barrier. (b) A representation of $1 \mathrm{D}$ subbands dispersion relations in nanowires. (c) The $1^{\text {st }} 1 \mathrm{D}$ subband and average transmission function, $T_{1}(E)$ and $T(E)$, across the square barrier formed in a InAs-InP-InAs heterostructure nanowire. The nanowire diameter is $60 \mathrm{~nm}$ and the InP length $L$ is $66 \mathrm{~nm}$.

\section{Reference:}

1. Levy-Leblond, J.-M. Elementary Quantum Models with Position-Dependent Mass. Eur. J. Phys. 1992, 13 (5), 215-218.

2. Davies, J. The Physics of Low-Dimensional Semiconductors. Cambridge University Press. 1998, p 4.

3. Faria Junior, P. E.; Campos, T.; Bastos, C. M. O.; Gmitra, M.; Fabian, J.; Sipahi, G. M. Realistic Multiband K.p Approach from Ab Initio and Spin-Orbit Coupling Effects of InAs and InP in Wurtzite Phase. Phys. Rev. B 2016, 93 (23), 235204. 